\title{
Wettability effect on flow boiling characteristics within micro-passages
}

\author{
Konstantinos Vontas ${ }^{1}$, Manolia Andredaki ${ }^{1}$, Anastasios Georgoulas ${ }^{1}$, Nico Miché ${ }^{1}$ and $^{\text {Marco Marengo }}{ }^{1}$ \\ ${ }^{1}$ Advanced Engineering Centre, School of Computing Engineering and Mathematics, University of Brighton, Lewes Road, \\ BN2 4GJ, Brighton, East Sussex, U.K \\ k.vontas@brighton.ac.uk; m.andredaki@brighton.ac.uk; a.georgoulas@brighton.ac.uk; n.d.d.miche@brighton.ac.uk; \\ m.marengo@brighton.ac.uk
}

\begin{abstract}
A numerical investigation on the effect of wettability characteristics on a single bubble growth during saturated flow boiling conditions within a microchannel, is conducted in the present paper. The numerical simulations are conducted with the open-source toolbox OpenFOAM, utilising a user-enhanced Volume OF Fluid (VOF) solver. The proposed solver enhancements involve a treatment for spurious velocities dampening (a well-known defect of VOF methods), an improved dynamic contact angle treatment to accurately account for wettability effects as well as the implementation of a phase-change model in the fluid domain, accounting for conjugate heattransfer with a solid domain. The predictions of the simulations show that the local Nusselt number $(\mathrm{Nu})$ is more depended on wettability characteristics for low heat fluxes, and less dependent on higher heat fluxes. In more detail, it seems that the local, instantaneous heat transfer coefficient is higher for super-hydrophilic cases in comparison to hydrophilic. However, as the applied heat flux increases, hydrophilic and super-hydrophilic cases show a similar heat transfer enhancement with respect to the single-phase heat transfer in the considered micro-channel. Finally, superhydrophobic cases, show lower heat transfer performance with respect to the single-phase case. This is due to the fact that a vapour blanket is rapidly formed immediately after the nucleation, acting as an insulator of the heated solid surface.
\end{abstract}

Keywords: Flow boiling, microchannel, multiphase flow, VOF, conjugate heat transfer.

\section{Introduction}

Due to the continuously increasing performance requirements and miniaturisation trends of micro-electronic components, such as computer chips and high-power electronics, their efficient thermal management has become a challenging issue. In more detail, very high heat flux values need to be dissipated, within really small spaces. It has been reported [1] that by 2026, the average heat flux that needs to be dissipated from computer chips is expected to reach values in the range of 2-4.5 MW/m² with local hot spots in the range of 12-45 MW/m². Similarly, Insulated Gate Bipolar Transistor (IGBT) modules, at the chip level, might reach heat flux values in the range of $6.5-50 \mathrm{MW} / \mathrm{m}^{2}$. Conventional cooling methods, such as air cooling through fans or pumped liquid cooling, cannot cope with such high heat dissipation demands. Flow boiling heat transfer within microchannels has been recognised experimentally as one of the most efficient thermal management solutions for such high-power density electronic components, dissipating heat fluxes in the order of $\mathrm{MW} / \mathrm{m}^{2}$ [2], [3]. However, cooling of micro-electronics utilising flow boiling within micro-passages is not yet commercially available and it is still limited to laboratory applications. The main reason for this, is the lack of accurate design correlations as well as the lack of understanding of several underpinned principles and mechanisms. Karayiannis and Mahmoud [4] recently reported that there are still open fundamental research aspects and issues. One of these is the effect of the solid surface characteristics on the underpinned bubble dynamics and heat transfer. In more detail, the solid surface manufacturing method, the application of surface coatings to enhance heat transfer and the solid surface material properties, might all significantly affect the underpinned phenomena and mechanisms.

In particular the solid surface wettability can be directly or indirectly linked with the aforementioned aspects. Previous experimental investigations have identified that the surface wettability characteristics have a significant impact on the global heat transfer coefficient [5], [6], [7]. However, in such experimental investigations in order to alter the surface wettability characteristics different solid materials are used, or the channel surfaces are treated accordingly through the application of coatings [8] that alter the structure, properties and the chemical composition of the surfaces. This might lead to the alteration of other factors, apart from the solid/liquid/vapour contact angle, that can additionally affect the flow boiling characteristics, 
such as the nucleation site density and the solid surface thermal response. Therefore, the effect of wettability cannot be isolated and directly identified and quantified, utilising experimental measurements. The present numerical investigation aims to isolate, identify and quantify the effect of wettability on the transient local heat transfer characteristics by "virtually" altering the advancing and receding contact angles for the case of an isolated bubble nucleus that grows within a microchannel in saturated flow boiling conditions. Advancing and receding contact angle values corresponding to super-hydrophilic, hydrophilic as well as superhydrophobic channels are simulated for this purpose, for a constant mass flow rate but for different values of applied heat flux.

\section{Numerical Method}

\section{1. Governing Equations}

The numerical simulations of the present paper are conducted with the open-source toolbox OpenFOAM, utilising a userenhanced Volume OF Fluid (VOF) solver. The proposed solver enhancements involve a treatment for spurious velocities dampening (a well-known defect of VOF methods), an improved dynamic contact angle treatment to accurately account for wettability effects as well as the implementation of a phase-change model in the fluid domain, accounting for conjugate heattransfer with a solid domain. In this section, the governing equations for mass, momentum, energy, and volume fraction are presented. It should be mentioned that liquid and vapour phases are both treated as incompressible, Newtonian fluids. This solver has been extensively validated in the past against various cases of adiabatic and diabatic bubble and droplet dynamics in comparison with available analytical solutions as wells as experimental measurements. Further details can be found in [9]-[12].

The mass conservation equation is given as:

$$
\nabla \cdot(\rho \vec{U})=\dot{\rho}
$$

where $\vec{U}$ is the fluid velocity and $\rho$ is the bulk density. The source term on the right-hand side accounts for the phase change. The conservation of momentum is given by the following equation:

$$
\frac{\partial}{\partial \mathrm{t}}(\rho \overrightarrow{\mathrm{U}})+\nabla \cdot(\rho \overrightarrow{\mathrm{U}} \overrightarrow{\mathrm{U}})-\nabla \cdot\left\{\mu\left[\nabla \overrightarrow{\mathrm{U}}+(\nabla \overrightarrow{\mathrm{U}})^{\mathrm{T}}\right]\right\}=-\nabla \mathrm{p}+\overrightarrow{\mathrm{f}}_{\mathrm{ST}}+\overrightarrow{\mathrm{f}}_{\mathrm{g}}
$$

where $\mathrm{p}$ is the pressure and $\mu$ is the bulk dynamic viscosity. The momentum source terms on the right-hand side of the equation account for the effects of surface tension and gravity, respectively. The surface tension term is modelled according to the classical approach of Brackbill et al. [13]. The conservation of energy balance is given by the following equation:

$$
\frac{\partial}{\partial \mathrm{t}}\left(\rho \mathrm{c}_{\mathrm{p}} \mathrm{T}\right)+\nabla \cdot\left(\overrightarrow{\mathrm{U}} \rho \mathrm{c}_{\mathrm{p}} \mathrm{T}\right)-\nabla \cdot(\lambda \nabla \mathrm{T})=\dot{\mathrm{h}}
$$

where $c_{p}$ is the bulk heat capacity, $T$ the temperature field, and $\lambda$ is the bulk thermal conductivity. The source term on the right-hand side of the equation represents the contribution of the enthalpy of evaporation/condensation or else the cooling/heating associated with the latent heat of the phase-change. The volume fraction $\alpha$ is advected by the flow field by the following equation:

$$
\frac{\partial \alpha}{\partial \mathrm{t}}+\nabla \cdot(\alpha \overrightarrow{\mathrm{U}})-\nabla \cdot\left(\alpha(1-\alpha) \overrightarrow{\mathrm{U}}_{\mathrm{r}}\right)=\frac{\dot{\rho}}{\rho} \alpha
$$


Interface sharpening is very important in simulating two-phase flows of two immiscible fluids. In OpenFOAM the sharpening of the interface is achieved artificially by introducing the extra compression term $\nabla \cdot\left(\alpha(1-\alpha) \overrightarrow{\mathrm{U}}_{\mathrm{r}}\right)$ in Equation (4). $\vec{U}_{\mathrm{r}}$ is an artificial compression velocity. The source term on the right-hand side of the Equation (4) is needed because, due to the local mass source terms, the velocity field is not free of divergence. Finally, the bulk fluid properties $\gamma$ are computed as the averages over the liquid $\left(\gamma_{1}\right)$ and vapour $\left(\gamma_{v}\right)$ phases, weighted with the volume fraction $\alpha$, i.e. $\gamma_{=} \alpha \gamma_{1}+(1-\alpha) \gamma_{v} . S$ mentioned previously, the VOF-based solver that is used in the present investigation has been modified accordingly in order to account for an adequate level of spurious currents suppression. More details on the proposed development and validation as well as on the proposed improved VOF method can be found in the paper by Georgoulas et al [9].

The conservation of energy equation in the solid domains is defined as:

$$
\frac{\partial}{\partial \mathrm{t}}\left(\rho_{s} \mathrm{c}_{\mathrm{ps}} \mathrm{T}\right)=\nabla \cdot\left(\lambda_{s} \nabla \mathrm{T}\right)
$$

The coupling at the interface between the solid region and fluid region is achieved iteratively through the following conditions:

$$
\begin{aligned}
& T_{f}=T_{s} \\
& \lambda_{f} \frac{\partial T_{f}}{\partial n}=\lambda_{s} \frac{\partial T_{s}}{\partial n}
\end{aligned}
$$

where $T_{f}$ is the temperature at the fluid side of the conjugate heat transfer boundary, $T_{s}$ is the temperature at the solid side of the conjugate heat transfer boundary, $\lambda_{f}$ is the thermal conductivity of the fluid domain and $\lambda_{s}$ is the thermal conductivity of the solid domain.

The local heat transfer $\mathrm{h}(\mathrm{x})$ coefficient can be calculated from the numerical simulation results as follows:

$$
h(x)=\frac{q^{\prime \prime}}{\left(T_{w}(x)-T_{s a t}\right)}
$$

where $\mathrm{x}$ represents the central longitudinal axis of the conjugate heat transfer boundary, $\mathrm{q}^{\prime \prime}$ is the applied heat flux in $\mathrm{W} / \mathrm{m}^{2}$ at the bottom surface of the solid domain, $\mathrm{T}_{\mathrm{w}}(\mathrm{x})$ is the temperature along the middle longitudinal line of the conjugate heat transfer boundary and $\mathrm{T}_{\text {sat }}$ is the saturation temperature (please see Fig.1). At this point it should be mentioned that saturated flow boiling conditions are used in all cases. The heat transfer coefficient can be expressed also in non-dimensional terms with the Nusselt number which is the ratio of the convective heat transfer to the conductive heat transfer. The following relationship is used for the calculation of the $\mathrm{Nu}$ number:

$$
N u=\frac{h D_{h}}{\lambda_{l}}
$$

where $D_{h}$ is the hydraulic diameter of the channel and $\lambda_{l}$ is the thermal conductivity of the liquid.

\section{2. Phase Change Model}

The utilized phase change model that was implemented in the improved OpenFOAM VOF solver that is used in the present investigation, will be described briefly in this section. In the case of evaporation, the evaporating mass flux at the liquid - vapour interface $j_{\text {evap }}$ is calculated from the following equation: 


$$
\mathrm{j}_{\text {evap }}=\frac{\mathrm{T}_{\mathrm{int}}-\mathrm{T}_{\mathrm{sat}}}{\mathrm{R}_{\mathrm{int}} \mathrm{h}_{\mathrm{lv}}}
$$

where $T_{\text {int }}$ is the temperature of the interface, $T_{\text {sat }}$ is the saturation temperature, $R_{\text {int }}$ is the interfacial heat resistance and $h_{\text {lv }}$ is the latent heat of evaporation at the saturation temperature. The amount of liquid that evaporates is calculated locally and the resulting source term field is smeared over a few cells in order to avoid numerical instabilities. The evaporating mass is taken away on the liquid side of the interface and reappears on the vapour side, following the process originally suggested in the work of Hardt and Wondra [14]. Further details on the proposed development can be found in the work of Georgoulas et al. [15].

\section{3. Dynamic Contact Angle Treatment}

The utilised numerical simulation model includes also an implementation of the dynamic contact angle (DCA) model originally suggested by Kistler [16]. This implementation has been validated in the past against experiments available in the literature for droplet impact on flat surfaces with different wettability, and it was shown that it can predict accurately both the spreading and recoiling stages of the impacts. Detailed information can be found here [17]. Furthermore, the numerical model predictions using the proposed DCA treatment have been also recently validated against experiments of pool boiling in biphilic surfaces [18].

\section{Application of Numerical Model}

\section{1. Computational Geometry, Mesh and Boundary Conditions}

In Fig. 1 (a) the generated computational geometry, mesh and the applied boundary conditions are illustrated. As it can be seen the computational mesh has been generated by discretising the computational domain in two parts. The solid domain and the fluid domain. A uniform, structured computational mesh, consisting of hexahedral elements is utilised. After a mesh independence study, an element size of $2 \mu \mathrm{m}$ was selected. Due to space limitation indicative results from the mesh independency study are shown in Fig. 1 (c), for one of the cases for a particular time instance. The total number of cells for the solid domain is $2.025 \mathrm{M}$ and $6.75 \mathrm{M}$ for the fluid domain. The fluid domain dimensions (length, height and width) are $\mathrm{L}_{\mathrm{f}}$ $=1.2 \mathrm{~mm}, \mathrm{H}_{\mathrm{f}}=0.30 \mathrm{~mm}, \mathrm{~W}_{\mathrm{f}}=0.15 \mathrm{~mm}$ and the solid domain dimensions are $\mathrm{L}_{\mathrm{s}}=1.2 \mathrm{~mm}, \mathrm{H}_{\mathrm{s}}=0.09 \mathrm{~mm}, \mathrm{~W}_{\mathrm{s}}=0.15 \mathrm{~mm}$. Except from the inlet and outlet in the fluid domain and the bottom side of the solid domain, where a constant heat flux is applied, all the rest surfaces are considered to be adiabatic walls. At the solid walls, a no-slip velocity boundary condition was used with a fixed flux pressure boundary condition for the pressure values. Moreover, a dynamic contact angle boundary condition is imposed for the volume fraction field by assigning the maximum advancing and minimum receding contact angle values from sessile drop measurements, that are then used for the calculation of the dynamic apparent contact angle values during the computations. For the sidewalls of the fluid and solid domains, a zero gradient boundary condition was used for the temperature field. At the outlet, a fixed-value pressure boundary condition and a zero-gradient boundary condition for the volume fraction were used, while for the velocity values a special (combined) type of boundary condition was used that applies a zero-gradient when the fluid mixture exits the computational domain and a fixed value condition to the tangential velocity component, in cases that the fluid mixture enters the domain. Finally, a zero gradient boundary condition for the temperature field was also prescribed at the outlet boundary. For the inlet, a constant uniform velocity value was imposed as well as a fixed flux pressure condition. The volume fraction value was assigned as unity, as saturated liquid only enters from the inlet during the calculations. Hence, the temperature of the liquid at the inlet was also fixed at the saturation temperature. As mentioned earlier both the temperature values as well as the heat fluxes are coupled at the conjugate heat transfer interface between the solid and fluid domains for each calculation time step through an inner iteration process.

\section{2. Numerical Simulation Set-up and Process}

As shown in Figure 1(a) the considered configuration for the present parametric numerical investigation consists of a single microchannel with a heat flux q" applied at its bottom solid wall. The rest of the walls are considered to be insulated 
(i.e. adiabatic). Therefore, the generated 3D computational domain consists of a solid domain (representing the bottom solid wall of the channel) that is in contact with the fluid domain that corresponds to the considered micro-passage. All the simulations are performed in two main stages. During the first stage a specified heat flux is applied to the bottom surface of the solid domain, and a single-phase liquid flow is considered initially with saturated liquid flowing with a specified mass flow rate. This is run up to a point that a steady state condition is reached and both the hydrodynamic and thermal boundary layers, have been developed. The velocity of the liquid $U_{1}$ is constant and uniform through the inlet boundary and the flow is laminar. This first stage is common for all of the two-phase simulations (second stage) that correspond to a particular value of applied heat flux. At the second stage a small vapour nucleus (bubble seed) represented as a half-sphere with a radius of $20 \mu \mathrm{m}$ is patched on the conjugate heat transfer boundary (interface between the fluid and liquid domains) at a distance of $200 \mu \mathrm{m}$ from the channel inlet, at saturation temperature. Since this initial bubble seed is patched within the previously developed thermal boundary layer where the temperature is higher than the saturation temperature, boiling occurs at the meniscus (solid/liquid/vapour triple line) as well as at the parts of the liquid/vapour interface that is in contact with temperatures higher that the saturation temperature. It should be mentioned that for all of the simulations conducted for the present investigation the liquid and vapour phase properties are taken as these of Ethanol liquid and vapour at the saturation equilibrium point for a pressure of $\mathrm{P}_{\mathrm{sat}}=1 \mathrm{bar}$, which corresponds to a saturation temperature of $\mathrm{T}_{\mathrm{sat}}=351.05 \mathrm{~K}$ (REFPROP NIST software). Hence, the liquid and vapour densities where taken as $\rho_{\mathrm{l}}=736.78 \mathrm{~kg} / \mathrm{m}^{3}$ and $\rho_{\mathrm{v}}=1.63 \mathrm{~kg} / \mathrm{m}^{3}$, the liquid and vapour kinematic viscosities as $v_{1}=6.01 \times 10^{-7} \mathrm{~m}^{2} / \mathrm{s}$ and $v_{\mathrm{v}}=6.37 \times 10^{-6} \mathrm{~m}^{2} / \mathrm{s}$, the liquid and vapour thermal conductivities as $\lambda_{1}=0.15 \mathrm{~W} / \mathrm{mK}$ and $\lambda_{\mathrm{v}}=0.02 \mathrm{~W} / \mathrm{mK}$ and the liquid and vapour heat capacities as $c_{\mathrm{p}, \mathrm{l}}=3182 \mathrm{~J} / \mathrm{kgK}$ and $\mathrm{c}_{\mathrm{p}, \mathrm{v}}=1804 \mathrm{~J} / \mathrm{kgK}$. As for the solid domain stainless steel properties are used. In more detail the density of the solid domain is taken as $\rho=7840$ $\mathrm{kg} / \mathrm{m}^{3}$, the specific heat as $\mathrm{c}_{\mathrm{v}, \mathrm{s}}=500 \mathrm{~J} / \mathrm{kgK}$ and the thermal conductivity as $\lambda_{\mathrm{s}}=16.2 \mathrm{~W} / \mathrm{mK}$. The advancing $\left(\theta_{\mathrm{a}}\right)$ and receding $\left(\theta_{\mathrm{r}}\right)$ contact angles for the base case of the present parametric analysis were taken as these of Ethanol with a particular stainless-steel sample with roughness $\mathrm{Ra}=0.4 \mu \mathrm{m}$, having values of $19^{\circ}$ and $18^{\circ}$, respectively. In order to investigate the isolated effects of wettability in the bubble growth characteristics as well as its effect in the local heat transfer, in the rest of the runs the values of $\theta_{\mathrm{a}}$ and $\theta_{\mathrm{r}}$ were varied while all the rest fluid properties and conditions were kept unaltered. The values that were selected for this purpose correspond to realistic contact angles that correspond to different combinations of Ethanol as well as other different working fluids (e.g. R-113, Glycerol and Water) with other uncoated or coated Stainless Steel samples of the same or different grades. In order to observe the effects of wettability at different heat fluxes, the overall analysis for all of the selected contact angle values was performed for three different values of applied heat flux $\left(q^{\prime \prime}=20,50\right.$, $100 \mathrm{KW} / \mathrm{m}^{2}$ ). The overall details regarding the wettability characteristics considered for each of the applied heat flux values in the numerical cases are summarised in Table 1. As it can be seen from the advancing and receding contact angle values three super-hydrophilic cases, two hydrophilic cases and one super-hydrophobic case, were considered for each of the three applied heat flux values. Therefore, a total of 18, 3D, transient, numerical simulations were performed. A High-Performance Computing (HPC) cluster was utilised for these runs. Each run required 100 computational cores. The duration of the computation for each of the three single phase simulations (one for each $q^{\prime \prime}$ value) was approximately 10 days, as seconds of real flow were required for a steady state condition to be reached. For the two-phase simulations the duration of the computation was only a couple of days since just a few milliseconds of real flow where required for the generated vapour bubble to reach the outlet of the channel. In Total 158,400 core-hours were utilized for the final runs that are presented in the paper. A variable calculation time step was utilised for the two-phase runs with the courant number kept constant at 0.5 . Hence the calculation time step was varied automatically ranging from $10^{-8}$ up to $10^{-6} \mathrm{~s}$.

Table 1: Considered wettability characteristics (these cases are considered for $\mathrm{q}^{\prime \prime}=20,50,100 \mathrm{KW} / \mathrm{m}^{2}$ ).

\begin{tabular}{|l|l|l|l|l|l|l|l|l|}
\hline Case & Equivalent Wettability & $\boldsymbol{\theta}_{\boldsymbol{a}}\left(\boldsymbol{(}^{\boldsymbol{}}\right)$ & $\boldsymbol{\theta}_{\boldsymbol{r}}\left(\boldsymbol{(}^{\boldsymbol{o}}\right)$ & Case & Equivalent Wettability & $\boldsymbol{\theta}_{\boldsymbol{a}}\left({ }^{\boldsymbol{o}}\right)$ & $\boldsymbol{\theta}_{\boldsymbol{r}}\left({ }^{\boldsymbol{o}}\right)$ \\
\hline I & $\begin{array}{l}\text { Ethanol with a particular Stainless } \\
\text { Steel of Ra=0.4 } \mu \mathrm{m}\end{array}$ & 0 & 0 & IV & $\begin{array}{l}\text { Glycerol with Stainless Steel } \\
316\end{array}$ & 49 & 0 \\
\hline II & R-113 with Stainless Steel AISI 316 & 16 & 5 & V & Water on Stainless Steel 316 & 70 & 40 \\
\hline III & $\begin{array}{l}\text { Ethanol with Stainless Steel AISI } \\
316\end{array}$ & 19 & 8 & VI & $\begin{array}{l}\text { Water with coated Stainless } \\
\text { Steel 316 }\end{array}$ & 162 & 159 \\
\hline
\end{tabular}



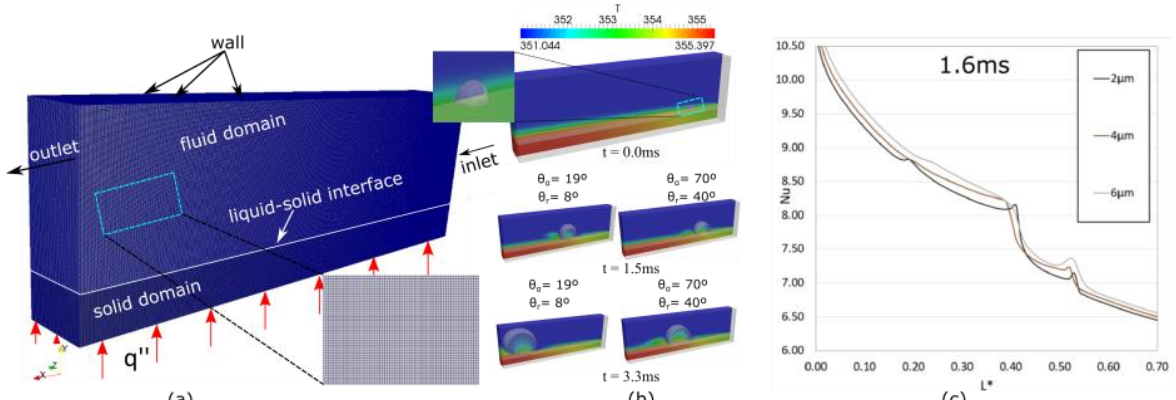

(a)

(b)

(c)

Fig. 1: (a) Computational domain, boundary conditions and mesh details, (b) spatial and temporal evolution of the vapour bubble for q" $=20 \mathrm{~kW} / \mathrm{m} 2$ and for wettability cases III and V (please see Table 1) and (c) mesh independency study results for Nu number distribution along the channel bottom boundary for Case III (Table 1) and time instance 1.6ms.

\section{Numerical Simulation Results}

The spatial and temporal evolution of the vapour bubble for Cases III and V for an applied heat flux value of $q$ "' = $20 \mathrm{~kW} / \mathrm{m}^{2}$, is depicted in Fig. 1(b). The grey surface represents the liquid/vapour interface, while the coloured contours in a clip section that extends from one side of the channel up to its middle plane, reveal the developed temperature fields in both the fluid and solid regions of the computational domain. It is evident that the variation of the advancing and receding contact angle values (i.e. wettability) has a quite significant effect in the resulting bubble growth dynamics. In both cases the bubble grows due to evaporation at the vapour/liquid/solid line, but it does not detach from the channel wall but instead it slides towards the outlet of the channel, maintaining a contact area with the heated wall. Moreover, as it can also be observed, in both cases due to the associated latent heat of evaporation as the bubble grows and slides along the solid wall the temperature of the solid is decreasing. From a qualitative/macroscopic point of view, in the more hydrophilic case (case III), and despite the fact that the flow rate and the applied heat flux are the same, the generated vapour bubble slides faster towards the outlet of the channel and it therefore creates a more significant disturbance of the thermal boundary layer that results in a situation where the bubble moving front is in contact with a higher region of super-heated liquid. Therefore, this creates a different overall evaporating interface region in each case that affects the bubble growth rate and therefore the overall heat transfer. However, in order to identify and quantify the effects of wettability on the local instantaneous heat transfer, in Figs. 2, 3 and 4 the Nusselt number is plotted over the dimensionless length $\left(\mathrm{L}^{*}=\mathrm{L}(\mathrm{x}) / \mathrm{L}_{\max }\right)$ of the considered microchannel for each case, along the central longitudinal axis of the conjugate heat transfer boundary, for the three different values of the applied heat flux and for time instances of $0.2 \mathrm{~ms}, 0.6 \mathrm{~ms}$ and $1.2 \mathrm{~ms}$ after the bubble nucleation, respectively. In all cases also the single-phase curve is used as a reference. In each diagram the corresponding time instances of the 3D bubble development is also superimposed (as in Fig 1(b)) for Cases I (super-hydrophilic), IV (hydrophilic) and VI (super-hydrophobic). It is observed, that for time $\mathrm{t}=0.2 \mathrm{~ms}$ (Fig. 2) and for a low values of applied heat flux (q" $=20 \mathrm{~kW} \mathrm{~m}^{-2}$ ), cases I-III (superhydrophilic) show a similar heat transfer rate over the length of the considered microchannel. Moreover, cases IV and V (hydrophilic cases) show also a similar behaviour but quite different than cases I-III. Finally, only case VI (superhydrophobic case) shows a completely different behaviour indicating a pick in the Nu number close to the inlet that drops below the single-phase value for the rest of the channel. It is also characteristic that as the applied heat flux increases the difference between the $\mathrm{Nu}$ versus $\mathrm{L}^{*}$ curves for cases I to $\mathrm{V}$, progressively diminish, showing a much higher heat transfer rate across almost the entire length of the channel than the single-phase case. However, case VI shows a progressively lower heat transfer rate than the single-phase case, across the channel length, as the applied heat flux increases. Exactly the same trend is observed also for the later time instances of $t=0.6 \mathrm{~ms}$ (Fig. 2) and $\mathrm{t}=1.2 \mathrm{~ms}$ (Fig. 3). A general conclusion that can be withdrawn from the above observations is that the solid surface wettability has a significant effect on the local heat transfer rates during flow boiling within micro-passages, for relatively low values of applied heat flux. However, for relatively high values (e.g. $100 \mathrm{~kW} \mathrm{~m}^{-2}$ ) of applied heat flux, the proposed effect is minimal. Finally, it is also evident that the use of superhydrophobic surfaces seems to have a negative effect in the heat transfer as for all of the examined time instances the super- 
hydrophobic surface shows a lower Nu number, for the majority of the channel length, in comparison to the single-phase case. This is due to the fact that in these cases, immediately after the bubble nucleation a vapour layer is formed that covers the entire heated wall, acting as an insulation due to the low thermal conductivity of the vapour phase.
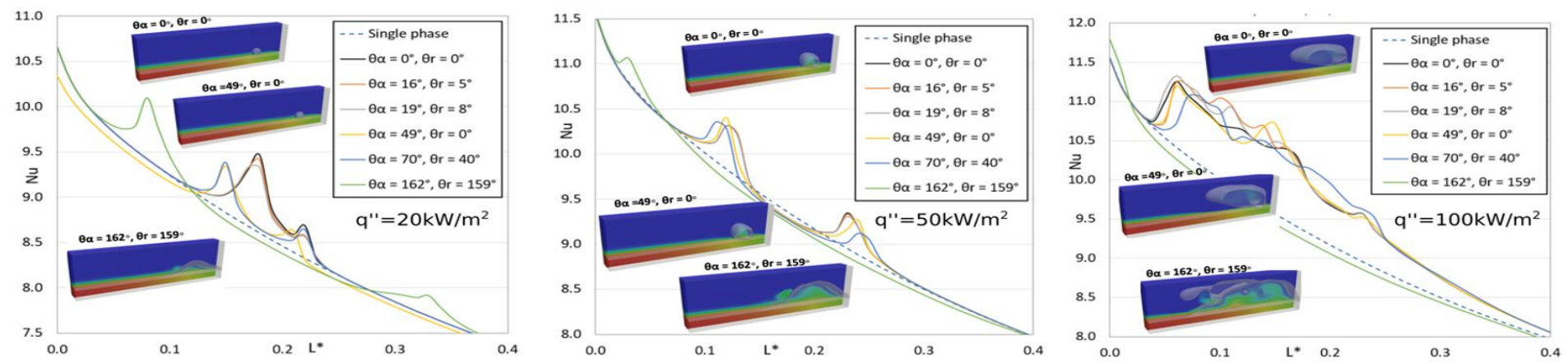

Fig. 2: Effect of wettability and heat flux on Nusselt number as a function of dimensionless length of microchannel for $\mathrm{t}=0.2 \mathrm{~ms}$.
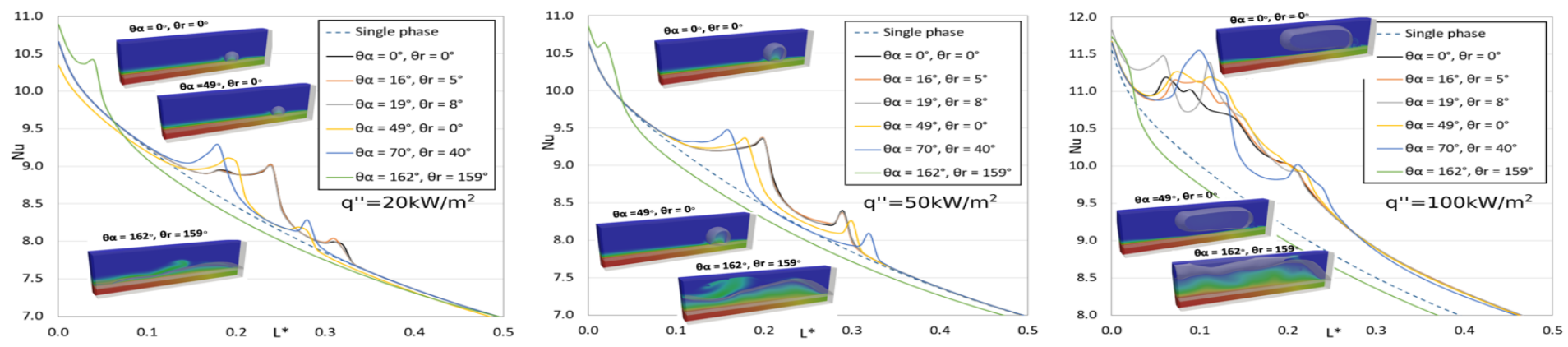

Fig. 3: Effect of wettability and heat flux on Nusselt number as a function of dimensionless length of microchannel at $\mathrm{t}=0.6 \mathrm{~ms}$.
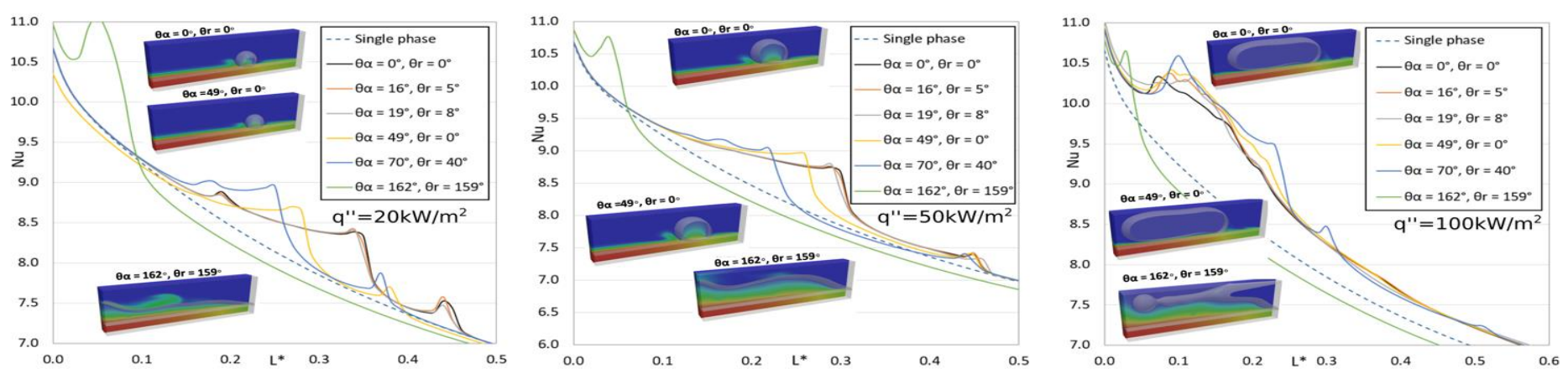

Fig. 4: Effect of wettability and heat flux on Nusselt number as a function of dimensionless length of microchannel at $\mathrm{t}=1.2 \mathrm{~ms}$.

\section{Conclusions}

In the present paper, a parametric numerical investigation has been performed aiming to identify and quantify the effect of wettability in the bubble growth characteristics for the case of isolated bubbles growing within heated microchannels, during saturated flow boiling conditions. From the overall analysis and presentation of the results, it can be concluded that for the considered micro-channel geometry, Capillary and Reynolds number, wettability can play an important role on the heat transfer rate behaviour, especially for low values of applied heat flux. However, the proposed effect seems to 
progressively diminish for progressively higher heat fluxes. As the heat flux increases, in general, the heat transfer performance progressively increases with respect to the single-phase case, for super-hydrophilic and hydrophilic cases. At relatively low heat fluxes, super-hydrophilic and hydrophilic cases show a quite different performance, with minimal differences between different cases in the super-hydrophilic and hydrophilic contact angle ranges. Finally, for all of the examined heat fluxes, super-hydrophobic cases present a lower performance even in comparison with the single-phase case that is used for reference.

\section{Acknowledgements}

This research was partially funded through the European Union's Horizon 2020 research and innovation programme (Marie Skłodowska Curie grant agreement No 801604), the UK's Engineering and Physical Science Research Council (grant EP/P013112/1), and the European Space Agency (ESA MAP CORA projects TOPDESS and ENCOM4).

\section{References}

[1] T. G. Karayiannis and M. M. Mahmoud, "Flow boiling in microchannels: Fundamentals and applications," Appl. Therm. Eng., vol. 115, pp. 1372-1397, Mar. 2017.

[2] A. Recinella and S. G. Kandlikar, "Enhanced Flow Boiling Using Radial Open Microchannels With Manifold and Offset Strip Fins," J. Heat Transfer, vol. 140, no. 2, pp. 21502-21509, Sep. 2017.

[3] K. P. Drummond et al., "A hierarchical manifold microchannel heat sink array for high-heat-flux two-phase cooling of electronics," Int. J. Heat Mass Transf., vol. 117, pp. 319-330, Feb. 2018.

[4] T. G. Karayiannis and M. M. Mahmoud, "FLOW BOILING IN MICRO-PASSAGES: DEVELOPMENTS IN FUNDAMENTAL ASPECTS AND APPLICATIONS," in 16th International Heat Transfer Conference, IHTC-16, 2018.

[5] H. Trieu Phan, N. Caney, P. Marty, S. Colasson, and J. Gavillet, "Flow Boiling of Water on Nanocoated Surfaces in a Microchannel," J. Heat Transfer, vol. 134, no. 2, Dec. 2011.

[6] T. Y. Liu, P. L. Li, C. W. Liu, and C. Gau, "Boiling flow characteristics in microchannels with very hydrophobic surface to super-hydrophilic surface," Int. J. Heat Mass Transf., vol. 54, no. 1-3, pp. 126-134, Jan. 2011.

[7] C. Choi, J. S. Shin, D. I. Yu, and M. H. Kim, "Flow boiling behaviors in hydrophilic and hydrophobic microchannels," Exp. Therm. Fluid Sci., vol. 35, no. 5, pp. 816-824, Jul. 2011.

[8] F. Yang, W. Li, X. Dai, and C. Li, "Flow boiling heat transfer of HFE-7000 in nanowire-coated microchannels," Appl. Therm. Eng., vol. 93, pp. 260-268, Jan. 2016.

[9] A. Georgoulas, P. Koukouvinis, M. Gavaises, and M. Marengo, "Numerical investigation of quasi-static bubble growth and detachment from submerged orifices in isothermal liquid pools: The effect of varying fluid properties and gravity levels," Int. J. Multiph. Flow, vol. 74, pp. 59-78, 2015.

[10] A. Georgoulas, M. Andredaki, and M. Marengo, "An Enhanced VOF Method Coupled with Heat Transfer and Phase Change to Characterise Bubble Detachment in Saturated Pool Boiling," Energies, vol. 10, no. 3, p. 272, Feb. 2017.

[11] E. Teodori, P. Pontes, A. Moita, A. Georgoulas, M. Marengo, and A. Moreira, "Sensible heat transfer during droplet cooling: Experimental and numerical analysis," Energies, vol. 10, no. 6, 2017.

[12] K. Vontas, M. Andredaki, A. Georgoulas, K. S. Nikas, and M. Marengo, "Numerical Investigation of Droplet Impact on Smooth Surfaces with Different Wettability Characteristics: Implementation of a dynamic contact angle treatment in OpenFOAM," in 28th European Conference on Liquid Atomization and Spray Systems, 2017.

[13] J. Brackbill, D. Kothe, and C. Zemach, "A continuum method for modeling surface tension," J. Comput. Phys., vol. 100, no. 2, pp. 335-354, Jun. 1992.

[14] S. Hardt and F. Wondra, "Evaporation model for interfacial flows based on a continuum-field representation of the source terms," J. Comput. Phys., vol. 227, no. 11, pp. 5871-5895, 2008.

[15] A. Georgoulas, M. Andredaki, and M. Marengo, "An enhanced VOF method coupled with heat transfer and phase change to characterise bubble detachment in saturated pool boiling," Energies, vol. 10, no. 3, 2017.

[16] S. F. Kistler, "In Hydrodynamics of Wetting," in Wettability, no. 1993, J. C. Berg, Ed. (Dekker, New York, 1993), 
1993, pp. 311-429.

[17] K. Vontas, M. Andredaki, A. Georgoulas, K. S. Nikas, and M. Marengo, "Numerical Investigation of Droplet Impact on Smooth Surfaces with Different Wettability Characteristics: Implementation of a dynamic contact angle treatment in OpenFOAM.," Proc. ILASS-Europe 2017. 28th Conf. Liq. At. Spray Syst., no. September, pp. 6-8, 2017.

[18] P. Pontes, R. Cautela, E. Teodori, A. Moita, A. Georgoulas, and A. L. N. Moreira, "Bubble dynamics and heat transfer on biphilic surfaces," in Proceedings of the 16th UK Heat Transfer Conference (UKHTC2019), 2019, p. 3. 